\title{
ARTICLE OPEN Super sensitivity and super resolution with quantum teleportation
}

\author{
J. Borregaard (D) , T. Gehring ${ }^{2}$, J. S. Neergaard-Nielsen ${ }^{2}$ and U. L. Andersen ${ }^{2}$
}

We propose a method for quantum enhanced phase estimation based on continuous variable (CV) quantum teleportation. The phase shift probed by a coherent state can be enhanced by repeatedly teleporting the state back to interact with the phase shift again using a supply of two-mode squeezed vacuum states. In this way a sequential protocol exhibiting both super-resolution and super-sensitivity can be obtained due to the coherent addition of the phase shift. The protocol enables Heisenberg-limited sensitivity and super-resolution given sufficiently strong squeezing. The proposed method could be implemented with current or near-term technology of CV teleportation.

npj Quantum Information (2019)5:16; https://doi.org/10.1038/s41534-019-0132-4

\section{INTRODUCTION}

Quantum correlations can be used in a number of ways to enhance metrological performance. ${ }^{1-4}$ Highly entangled states such as NOON and GHZ states can enable Heisenberg-limited sensitivity yielding a square root improvement with the number of probes over the standard quantum limit (SQL). ${ }^{5-7}$ This kind of improvement is particularly useful when probing fragile systems where photon damage limits the allowed number of probe photons. This can be the case in, e.g., measuring of biological systems ${ }^{8-10}$ or cold trapped atoms. ${ }^{11}$ While this effect has been demonstrated in experiments for small probe sizes, ${ }^{11-14}$ scaling up the size of the entangled states remains a technological barrier due to their fragility to loss and noise. Other strategies based on the more experimentally accessible squeezed vacuum states have also shown to beat the SQL in various settings. ${ }^{15-20}$ An alternative strategy is to perform multi-pass protocols with a single probe. This enables both Heisenberg-limited sensitivity and superresolution ${ }^{21}$ for phase estimation without entangled resources by applying the phase shift to the same probe multiple times. ${ }^{22-24}$ Its experimental demonstration was realized by surrounding the phase shift system with mirrors to measure a transversally distributed phase shift ${ }^{25}$ or an image ${ }^{26}$ with Heisenberg-limited sensitivity. While these approaches have demonstrated the effect of sub-shot noise scaling without entanglement, both demonstrations were based on post-selection, rendering the efficiency very low. In addition, the former demonstration could only measure a transversally distributed phase shift requiring a sample size much larger than the beam size. The key problem is that the number of passes through the sample needs to be carefully controlled. For mirror-based approaches, this may be obtained with fast integrated optical routing on the timescale of the roundtrip time between mirrors, which can be very challenging experimentally.

Here we propose a fundamentally different method based on quantum teleportation for realizing quantum enhanced phase measurements. The essence of our proposal is to repeatedly teleport back the probe to coherently apply the phase shift multiple times (see Fig. 1), thus effectively realizing a multi-pass protocol. This circumvents the need for physically redirecting a probe state to the same phase shift multiple times and allows to keep the entangled resources separate from the potentially lossy phase shifting system. Compared to the mirror-based approach, no fast optical routing is required and the setup is more flexible in not having to be confined in between mirrors. We describe how this protocol can be implemented with current technology of continuous variable teleportation using two-mode squeezed vacuum states and an initial coherent state as a probe.

\section{RESULTS}

In the general setup, we consider some initial probe in a state $\left|\psi_{0}\right\rangle$ which is subject to an unknown phase shift described by a unitary $U(\phi)=e^{i \phi \hat{n}}$, where $\hat{n}$ is the photon number operator. The goal is to estimate the phase $\phi$. After the interaction, an entangled state is used to teleport the output state $U(\phi)\left|\psi_{0}\right\rangle$ back to interact with the phase shift again. This process is then iterated $m$ times. If the teleportation is perfect, this would correspond to the transformation $\left|\psi_{0}\right\rangle \rightarrow(U(\phi))^{(m+1)}\left|\psi_{0}\right\rangle=U((m+1) \phi)\left|\psi_{0}\right\rangle$ of the input state where $m$ is the number of teleportations. By coherently applying the phase $(m+1)$ times, the signal can have both super-resolution and super-sensitivity since it will now depend on $(m+1) \phi$ instead of just $\phi .^{22,23}$

As a physical realization of this protocol, we consider the setup illustrated in Fig. 1 where consecutive two-mode squeezed vacuum states are supplied by interferring the output of two single-mode squeezed vacuum sources on a balanced beam splitter. ${ }^{27}$ One mode is delayed in a fiber and will subsequently be subject to an unknown phase shift described by the unitary $U(\phi)$. Feedback based on previous measurements is applied before the phase shift. This feedback can be implemented by mixing in an auxiliary laser field using a high transmission beam splitter. ${ }^{27}$ The delay $(T)$ is chosen such that the phase shifted mode is interfered with the first mode of the subsequent two-mode squeezed

\footnotetext{
${ }^{1}$ QMATH, Department of Mathematical Sciences, University of Copenhagen, Universitetsparken 5, 2100 Copenhagen, Denmark and ${ }^{2}$ Center for Macroscopic Quantum States (bigQ), Department of Physics, Technical University of Denmark, Fysikvej, 2800 Kgs. Lyngby, Denmark

Correspondence: J. Borregaard (jborregaard@math.ku.dk)
}

Received: 27 July 2018 Accepted: 21 January 2019

Published online: 07 February 2019 
(a)

(b)

(c)

(d) (e) (f) (g)

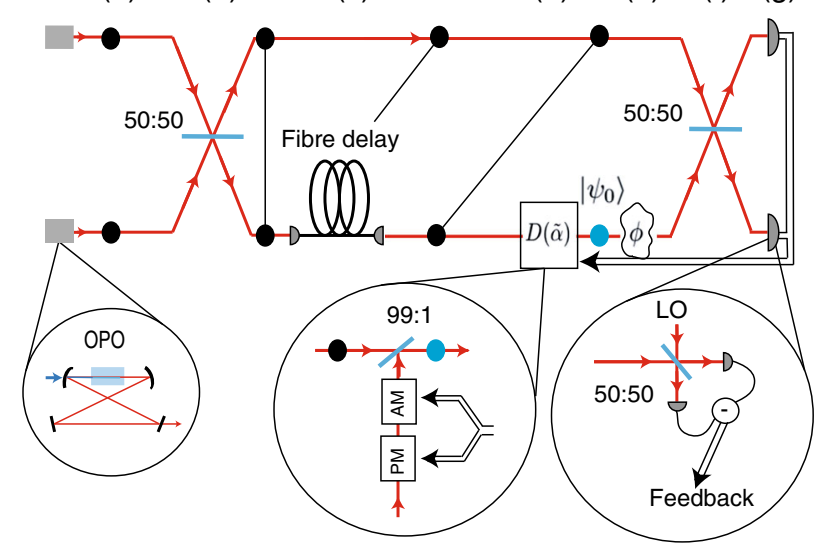

Fig. 1 Sketch of the setup. Two optical parametric oscillators (OPO) output single-mode squeezed vacuum states, which can be viewed as a consecutive train of independent squeezed temporal modes illustrated by black dots (a). Interfering the modes on a 50:50 beam splitter results in two-mode squeezed vacuum states shown as connected black dots (b). The bottom modes are input to a fiber delay so they coincide in time with the top mode of the subsequent pair (c). The bottom mode is subject to feedback based on previous measurements (d). This feedback can be implemented by mixing in an auxiliary laser subject to amplitude (AM) and phase (PM) modulation using a high transmission beam splitter (here 99:1). The feedback is part of teleporting back the initial probe state $\left|\psi_{0}\right\rangle$. The teleported state undergoes a phase shift $U(\phi)$ (e) before being mixed with the top mode of a two-mode squeezed vacuum pair on a 50:50 beam splitter (f). After the beam splitter, the quadratures of the output states are measured with homodyne detection as part of the repeated teleportation protocol giving the classical feedback shown with double lines $(\mathbf{g})$

vacuum state on a balanced beam splitter before measurement. The setup, which is similar to a Mach-Zehnder interferometer with feedback, is inspired by ref. ${ }^{28}$ where the generation of continuous variable (CV) cluster states is demonstrated. As demonstrated in ref. ${ }^{28}$, the squeezed light can be produced using optical parametric oscillation (OPO). Binning the temporal modes such that $T$ is larger than the inverse bandwidth of the OPO results in independent squeezed temporal modes. We choose the measurements and the feedback such that the CV teleportation protocol of ref. ${ }^{29}$ is realized. In this teleportation protocol, the momentum quadrature of one of the output modes and the position quadrature of the other is measured, which can be achieved with homodyne detection in a single-shot measurement. The feedback consists of quadrature displacement based on the measurement outcomes. For perfect teleportation, infinitely many photons are, in principle, needed in the two-mode squeezed vacuum states. The number of photons actually obtaining the phase shift will nonetheless only depend on the initial input state. For situations where the phase shift is obtained by interaction with a photosensitive system, ${ }^{8-11}$ the effective number of probe photons interacting with the system is the limited resource. This number will be $\sim(m+1) n_{0}$ where $n_{0}$ is the number of photons in the initial state. We will show that Heisenberg-limited sensitivity in terms of probe photons can be reached with a simple coherent state as input state. Furthermore, the phase resolution can be enhanced by a factor of $m+1$.

We consider a coherent state $|-i a\rangle$ as the initial probe state $\left|\psi_{0}\right\rangle$ $(a \in \mathbb{R})$. In the setup of Fig. 1, we can input the initial state by displacing the initial vacuum mode of the lower arm using the feedback laser. After the interaction of $U(\phi)$, the state will be $\left.-i a e^{i \phi}\right\rangle$. This state is now teleported back to the second mode of the first two-mode squeezed vacuum state following the CV protocol of ref. ${ }^{29}$ The two-mode squeezed vacuum state has squeezing parameter $r$ such that $\left\langle\left(\hat{x}_{2}-\hat{x}_{3}\right)^{2}\right\rangle=e^{-2 r} / 2$, where $\hat{x}_{2}$, $\hat{x}_{3}$ are the position quadratures for the two modes. The first mode of the two-mode squeezed vacuum state is mixed with the probe state on the balanced beam splitter before measurement. The output modes of the beam splitter have position quadratures $\hat{x}_{1}^{\prime}=$ $\left(\hat{x}_{1}+\hat{x}_{2}\right) / \sqrt{2}$ and $\hat{x}_{2}^{\prime}=\left(\hat{x}_{1}-\hat{x}_{2}\right) / \sqrt{2}$ with similar expressions for the momentum quadratures. Here $\hat{x}_{1}$ is the position quadrature of the probe state. The quadratures $\hat{p}_{1}^{\prime}$ and $\hat{x}_{2}^{\prime}$ are measured giving measurement outcomes $\left\{p_{1}^{\prime}, x_{2}^{\prime}\right\}$. The feedback then implements the displacements $\hat{x}_{3} \rightarrow \hat{x}_{3}^{\prime}=\hat{x}_{3}+g_{x} \sqrt{2} x_{2}^{\prime}$ and $\hat{p}_{3} \rightarrow \hat{p}_{3}^{\prime}=\hat{p}_{3}+g_{p} \sqrt{2} p_{1}^{\prime}$, which concludes the teleportation protocol of ref. ${ }^{29}$

The feedback displaces the quadratures such that the teleported state, $\psi_{1}$ will be close to $\left|-i a e^{i \phi}\right\rangle$. The quality of the teleportation will depend on the amount of squeezing contained in the two-mode squeezed vacuum state and the feedback strength quantified by the gains $g_{x}$ and $g_{p}$. In the limit of high squeezing, perfect teleportation is obtained for $g_{x}=g_{p}=1$. The protocol now repeats itself $m$ times corresponding to $m$ teleportations being performed. At the end of the protocol, the squeezed light sources should be switched off such that the final teleported state $\left|\psi_{\mathrm{m}}\right\rangle$ is not mixed with any two-mode squeezed states before measurement. The teleported state $\left|\psi_{\mathrm{m}}\right\rangle$ is subject to the phase shift resulting in state $\left|\psi_{\mathrm{m}}^{\prime}\right\rangle=U(\phi)\left|\psi_{\mathrm{m}}\right\rangle$. This state is split by the 50:50 beams splitter (with vacuum in the other input port) and the position quadratures of the output modes are measured and classically added with equal weight of $1 / \sqrt{2}$. This is equivalent to measuring the position quadrature $\left(\hat{x}_{m}^{\prime}\right)$ of $\left|\psi_{m}^{\prime}\right\rangle$ before the beam splitter and we can therefore simply consider this situation. Assuming gains of $g_{x}=g_{p}=1$, the mean and variance of $\hat{x}_{m}^{\prime}$ is

$\left\langle\hat{x}_{m}^{\prime}\right\rangle=\left\langle\psi_{m}^{\prime}\left|\hat{x}_{m}^{\prime}\right| \psi_{m}^{\prime}\right\rangle=a \sin ((m+1) \phi)$

$\operatorname{Var}\left(\hat{x}_{m}^{\prime}\right)=\left\langle\psi_{m}^{\prime}\left|\left(\left(\hat{x}_{m}^{\prime}\right)^{2}-\left\langle\hat{x}_{m}^{\prime}\right\rangle^{2}\right)\right| \psi_{m}^{\prime}\right\rangle=\frac{1+2 m e^{-2 r}}{4}$.

It is clear from Eq. (1) that the signal exhibits super-resolution in $\phi$ by a factor of $(m+1)$. The sensitivity of the measurement can be quantified $a s^{6}$

$\sigma_{m}=\frac{\sqrt{\operatorname{Var}\left(\hat{x}_{m}^{\prime}\right)}}{\left|\delta\left\langle\hat{x}_{m}^{\prime}\right\rangle / \delta \phi\right|}=\frac{\sqrt{1+2 m e^{-2 r}}}{2(m+1) a|\cos ((m+1) \phi)|}$.

Note that the sensitivity exhibits a linear decrease in the number of teleportations $m$ as long as $|\cos ((m+1) \phi)| \approx 1$ and the squeezing is sufficiently strong such that $2 m e^{-2 r} \ll 1$. For a classical (SQL) strategy with $m$ independent coherent states $|-i a\rangle$, the sensitivity would have a scaling of $\propto 1 /(\sqrt{m} a)$. The average number of probe photons, $n_{j}$ contained in the state $\left|\psi_{j}\right\rangle$ is

$n_{j}=a^{2}+j e^{-2 r}$,

thus the total average number of probe photons that have interacted with the phase shift system will be

$n_{\text {total }}=\sum_{j=0}^{m} n_{j}=(m+1) a^{2}+\frac{1}{2} m(m+1) e^{-2 r}$.

If the coherent state contains one photon $(a=1)$ on average, we have that $n_{\text {total }}=(m+1)\left(1+\frac{1}{2} m e^{-2 r}\right)$ and the sensitivity is

$\sigma_{m}=\frac{\sqrt{\left(1+\frac{1}{2} m e^{-2 r}\right)^{2}\left(1+2 m e^{-2 r}\right)}}{2 n_{\text {total }}|\cos ((m+1) \phi)|}$.

Thus, if $m e^{-2 r} \ll 1$, the sensitivity exhibits Heisenberg scaling in the number of probe photons for $|\cos ((m+1) \phi)| \approx 1$. This sensitivity is similar to what could be obtained using NOON states of $(m+1)$ photons and expresses the ultimate scaling allowed by quantum mechanics. ${ }^{1}$ 
(a)

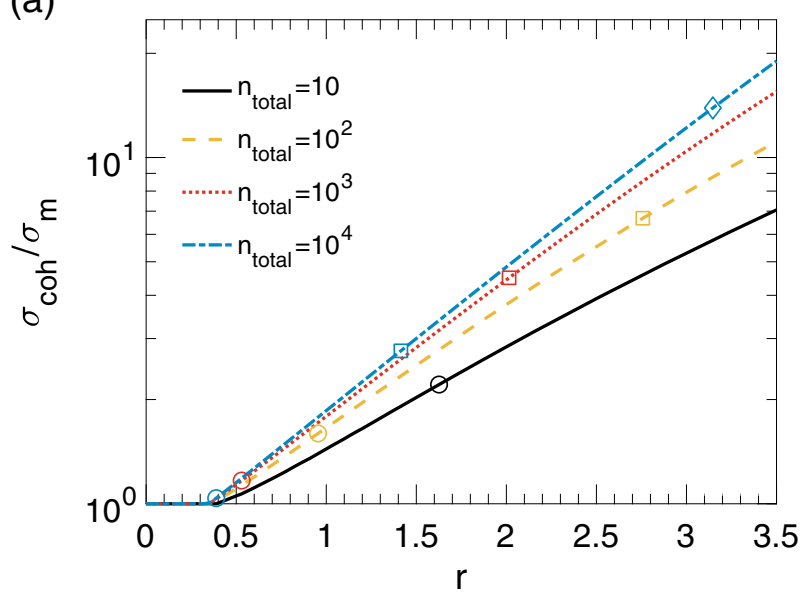

(b)

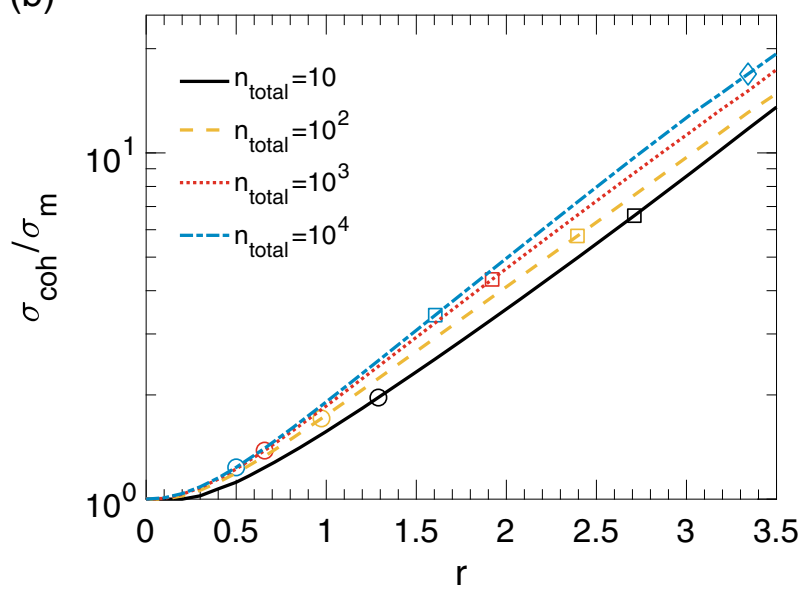

Fig. 2 Performance for finite squeezing. Maximum gain in sensitivity by using the teleportation scheme compared to a classical coherent state protocol for limited amount of squeezing $(r)$ and fixed average number of total photons $n_{\text {total }}$. We have assumed that $\mid \cos ((m+1)$ $\phi) \mid \approx 1$. The performance is better for high $n_{\text {total }}$ because here the photons added to the probe states by imperfect teleportation have smaller weight compared to the extra noise added to the quadrature. We have assumed gains of $g_{x}=g_{p}=1$ in (a) while we have numerically optimized the gains in (b). It is seen that in the limit $n_{\text {total }} \lesssim e^{2 r}$ the optimal gains are different from $g_{x}=g_{p}=1$. The optimal number of teleportations $m$ found in the optimizations are indicated with circles, squares and diamonds. These indicate the transitions to $m \geq 10,100$, and 1000 , respectively, on the curves

\section{Limited squeezing}

One of the dominant experimental limitations of the proposed protocol will arguably be the amount of squeezing in the twomode squeezed vacuum states. This will limit how many teleportations can be performed before the extra noise from the imperfect teleportations will dominate the signal. We therefore consider what the optimum strategy is given a constraint on the amount of squeezing. In addition, we also limit the total average number of photons that can interact with the phase shift system. We then optimize over the number of teleportations $m$ and the size of the coherent probe state $a$ to find the strategy that provides the maximum sensitivity for these limitations. Furthermore, we also allow for arbitrary gains $g_{x}$ and $g_{p}$. The result of the optimization is shown in Fig. 2 where we illustrate the performance relative to a standard coherent state protocol with matched average photon number. For such an approach, the sensitivity is simply $\sigma_{\text {coh }}=1 /\left(2 \sqrt{n_{\text {total }}}|\cos (\phi)|\right)$ where $n_{\text {total }}$ is the average number of probe photons. For $|\cos (\phi)| \approx 1$, the coherent state approach exhibits sensitivity at the SQL. Figure 2 shows the two effects of the imperfect teleportation; noise is added in the $\hat{x}$-quadrature (see Eq. (2)) and more photons are added to the probe state (see Eq. (5)). In the minimization, the error from the extra photons added by an imperfect teleportation has smaller weight for higher $n_{\text {total }}$. In the limit where $n_{\text {total }} \gg e^{2 r}$, the enhancement is $\sim e^{r} / \sqrt{2}$ and equal gains of $g_{x}=g_{p}=1$ are optimal. This is the limit where the extra photons added to the probe state do not have any significant effect on the optimum performance. We note that a similar enhancement in sensitivity could be obtained by using a squeezed coherent state as probe. $^{15,30}$ For such protocols, the squeezed photons, however, interact with the phase shift system, which is not the case here. Consequently, our protocol also works in the limit $n_{\text {total }} \ll e^{2 r}$ where an enhancement of $\sim\left(n_{\text {total }} e^{2 r} / 2\right)^{\frac{1}{4}}$ can be obtained for $g_{x}$ $=g_{p}=1$. Note that our numerical optimization shows that larger enhancement can also be obtained for optimized gains in this limit (see Fig. 2b). Finally, we note that no enhancement is possible when $r \lesssim 0.35$ for $g_{x}=g_{p}=1$ since the extra amplitude noise added by the teleportation cancels the gain in the signal (see Eq. (3)). For optimized gains, however, there can be a small enhancement.

Photon loss

One of the technological challenges of using highly entangled quantum states for enhanced phase measurements is that they are very fragile to losses. Multi-pass protocols share this fragility since losses grow exponentially with the number of passes through the sample. ${ }^{24}$ This means that if the losses are too high, the sensitivity enhancement of the multi-pass protocol proposed here will vanish. Note however that while approaches based on NOON states rely on single photon detection, this protocol is based on homodyne detection, which in practice is much more efficient. Since imperfect photon detection will add to the overall loss, this means that the effective loss may be substantially reduced with this protocol.

We investigate the performance of the proposed protocol in the presence of both loss acting on the probe state corresponding to a lossy phase shift system and loss acting on the two-mode squeezed vacuum states. Any (symmetric) detection loss would add directly to both of these losses. We model the losses with fictitious beam splitters where the unused output port is traced out. To model the lossy phase shift system, a fictitious beam splitter of transmission $\eta_{1}$ is inserted after the phase shift $U(\phi)$ (see Fig. 1). For the loss in the twomode squeezed vacuum state, fictitious beam splitters both with transmission $\eta_{2}$ are inserted for each of the modes. For simplicity, we have assumed equal losses for both modes. Assuming equal gains of $g_{x}=g_{p}=1$, the signal and sensitivity after $m$ teleportations for $\left\{\eta_{1}, \eta_{2}\right\}<1$ is

$\left\langle\hat{x}_{m}^{\prime}\right\rangle=a \eta_{1}^{\frac{m+1}{2}} \sin ((m+1) \phi)$

$\sigma_{m}=\frac{\sqrt{1+2 \eta_{1} \frac{1-\eta_{1}^{m}}{1-\eta_{1}}\left(\eta_{2} e^{-2 r}+1-\eta_{2}\right)}}{2(m+1) a \eta_{1}^{\frac{m+1}{2}}|\cos ((m+1) \phi)|}$.

As expected, the loss on the probe state $\left(\eta_{1}\right)$ enters in the expression for the sensitivity exponentially in $m$, while loss on the two-mode squeezed vacuum states $\left(\eta_{2}\right)$ only has a linear effect in $m$. The effect of $\eta_{2}<1$ on the sensitivity is equivalent to having a limited squeezing of $r_{\lim }=-\frac{1}{2} \ln \left(\eta_{2} e^{-2 r}+1-\eta_{2}\right)$. This also holds when considering the average number of total probe photons 
(a)

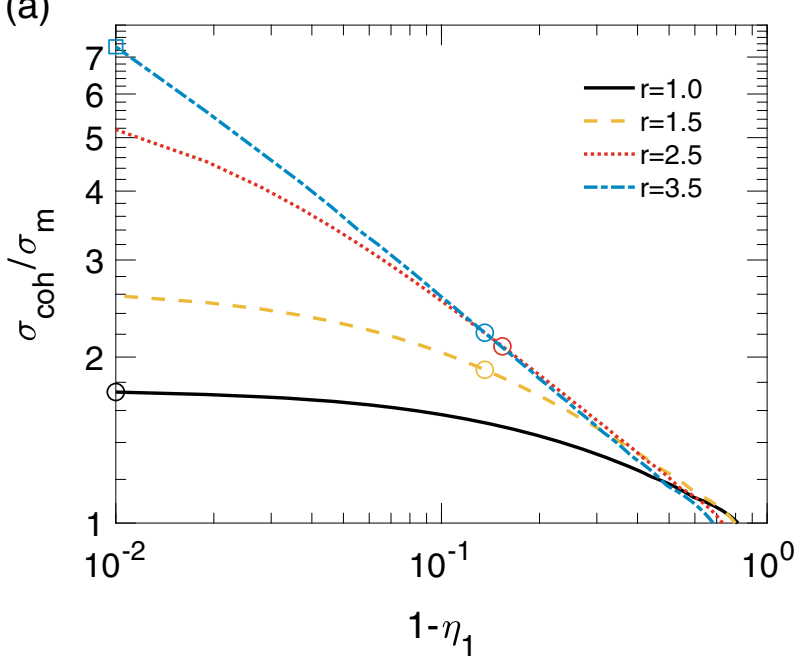

(b)

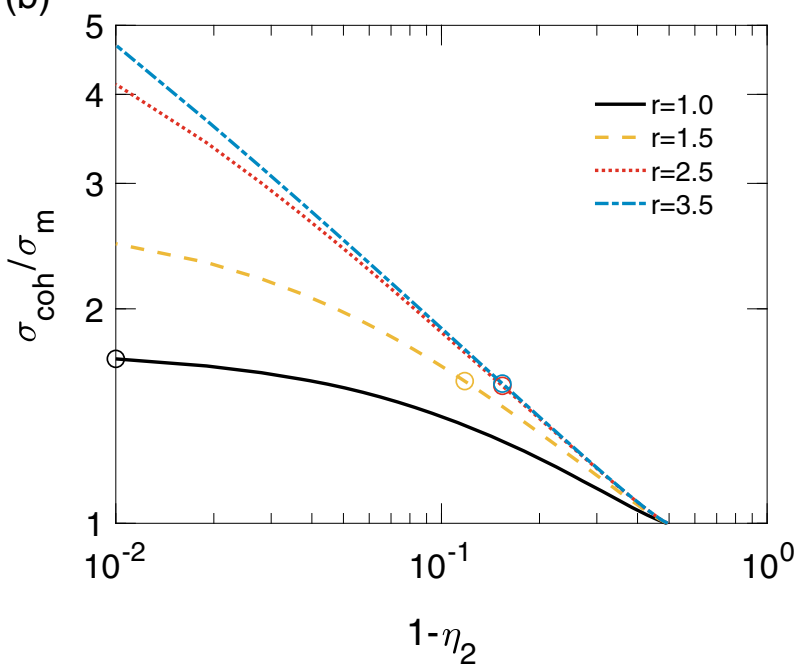

Fig. 3 Performance with loss. Maximum gain in sensitivity by using the teleportation scheme compared to a classical coherent state protocol for limited amount of squeezing $(r)$ and fixed average number of total photons $n_{\text {total }}=100$ in the presence of a loss on the probe state $\left(\eta_{1}<1, \eta_{2}=1\right)$ and $\mathbf{b}$ loss on the two-mode squeezed vacuum state $\left(\eta_{1}=1, \eta_{2}<1\right)$. The optimal number of teleportations $m$ decreases as the loss increases. The circles (squares) indicate the transition where $m \geq 10(m \geq 100)$. We have assumed that $\mid \cos ((m+$ 1) $\phi) \mid \approx 1$

incident on the phase shift system. For $m$ teleportations and gains of $g_{x}=g_{p}=1$, we have that

$n_{\text {total }}=\frac{1-\eta_{1}^{m+1}}{1-\eta_{1}} a^{2}+\frac{m\left(1-\eta_{1}\right)-\eta_{1}\left(1-\eta_{1}^{m}\right)}{\left(1-\eta_{1}\right)^{2}}\left(\eta_{2} e^{-2 r}+1-\eta_{2}\right)$.

Note that by taking the limit $\eta_{1} \rightarrow 1$ for $\eta_{2}=1$, Eqs. (7)-(9) reduce to Eqs. (1), (3) and (5). If excess noise on the squeezed states is included by mixing in thermal states of average photon number $\bar{n}$ instead of vacuum in the fictitious beam splitters $\left(\eta_{2}\right)$, one would have that $r_{\lim }=-\frac{1}{2} \ln \left(\eta_{2} e^{-2 r}+(1+2 \bar{n})\left(1-\eta_{2}\right)\right)$. We will assume that $\bar{n} \ll 1$ such that excess noise can be neglected. To see the effect of finite losses, we again compare the protocol to the simple coherent strategy for which $\sigma_{\text {coh }}=$ $1 /\left(2 \sqrt{\eta_{1} n_{\text {total }}}|\cos (\phi)|\right)$ in the presence of loss. The result of the optimization is shown in Fig. 3. While a small improvement was found by optimizing the gains, near optimal performance is reached for $g_{x}=g_{p}=1$. The error from losses in the two-mode squeezed vacuum state limits the gain in the same way as finite squeezing does for the lossless case. Consequently, when these losses dominate the error, the enhancement is $\sim 1 / \sqrt{2\left(1-\eta_{2}\right)}$ and no enhancement is possible for $\eta_{2} \sim 1 / 2$. When losses in the probe state limit the enhancement, the optimum performance is effectively found as a tradeoff between the $\sqrt{m+1}$ enhancement due to the teleportation and the exponential reduction due to the loss. As a result, we find that the enhancement is $\sim$ $\sqrt{2 /\left(3\left(1-\eta_{1}\right)\right)}$ and no enhancement is possible for $\eta_{1} \sim 1 / 3$. We note that while losses quickly reduce the enhancement, the scheme still exhibits enhanced sensitivity compared to the standard coherent state probe even for substantial losses.

\section{DISCUSSION}

In conclusion, we have shown how both super-sensitivity and superresolution can be obtained for an optical phase measurement using continuous variable quantum teleportation based on two-mode squeezed vacuum states. For negligible losses, the protocol can exhibit Heisenberg-limited sensitivity $(\sim 1 / N)$ for squeezing $2 \mathrm{Ne}^{-2 r} \ll 1$ and increase the resolution by a factor of $N$, where $N$ is the number of probes. While this is equivalent to the enhancement possible with $\mathrm{N}$-photon NOON states and single photon detection, ${ }^{6}$ the protocol proposed here relies on homodyne detection, which generally is more efficient than single photon detection. As a consequence of the super-resolution, the phase to be estimated should, in principle, be localized within a window of $1 / N$ to reach the Heisenberg limit as for a NOON or GHZ state approach. ${ }^{1,5}$ However, methods developed to estimate arbitrary phases, ${ }^{19,31}$ in particular, for NOON ${ }^{32}$ and GHZ states, ${ }^{33}$ might also be employed in a straightforward way to this scheme. The latter method uses $\mathrm{GHZ}$ states of different sizes in order to estimate the digits of the phase allowing for arbitrary phase estimation. ${ }^{33}$ The same technique could be employed here by operating with different number of teleportations before readout, which effectively corresponds to sending entangled states of varying sizes. We have also studied the effect of photon loss on the scheme both for loss in the two-mode squeezed vacuum states used for teleportation (limits the effective squeezing) and for loss on the probes corresponding to a lossy phase shift system. While loss quickly reduces the performance, the protocol may still provide super-sensitivity for loss on the order of several percent. For an effective squeezing of $13 \mathrm{~dB}$ $(r=1.5)$, a $6 \mathrm{~dB}$ enhancement of the sensitivity $\left(\sigma^{2}\right)$ may be obtained even with $10 \%$ loss in the phase shift system for $n_{\text {total }}=100$ using $m=12$ teleportations. For a more modest squeezing of $8 \mathrm{~dB}$ $(r=0.92)$, a $3.5 \mathrm{~dB}$ enhancement may be obtained under the same conditions with $m=6$ teleportations.

While the specific protocol studied here employed CV teleportation of a coherent state with two-mode squeezed vacuum states, the generic setup of teleporting back a probe state to interact with the phase shift system multiple times may be extended to other scenarios. In particular, our method can be easily extended to a multi-mode scheme to demonstrate Heisenberg-limited imaging. This can be realized by replacing the single-mode teleportation scheme with a multi-mode scheme in which multiple higher-order spatial modes are simultaneously teleported. ${ }^{34}$ Using such a multi-mode approach, sub-shot noise and eventually Heisenberg-limited microscopy can be realized. Non-Gaussian states such as photon subtracted two-mode squeezed states ${ }^{35}$ may also be considered for enhanced teleportation performance or different probe states providing better single-shot estimation. ${ }^{7}$ A discrete variable variant of the protocol could also be envisioned using one-dimensional cluster states emitted by single quantum emitters. ${ }^{36}$ Here every second qubit could probe the phase shift while the remaining qubits are used to teleport the phase information. 


\section{METHODS}

We evaluate the performance of the teleportation protocol using the Wigner functions ${ }^{37}$ of the photonic modes. The two-mode squeezed vacuum state has Wigner function

$W_{\text {sq }}\left(x_{2}, p_{2}, x_{3}, p_{3}\right)=\left(\frac{2}{\pi}\right)^{2} e^{-e^{-2 r}\left(\left(x_{2}+x_{3}\right)^{2}+\left(p_{2}-p_{3}\right)^{2}\right)} e^{-e^{2 r}\left(\left(p_{2}+p_{3}\right)^{2}+\left(x_{2}-x_{3}\right)^{2}\right)}$,

with $\left(x_{2}, p_{2}\right)$ and $\left(x_{3}, p_{3}\right)$ describing the position and momentum quadratures of the two modes, respectively. Photon loss and excess noise is included by mixing both modes with thermal fields of average photon number $\bar{n}$ on fictitious beam splitters of transmission $\eta_{2}$ (assumed equal for both modes). The input modes before the beam splitters are described by the Wigner function

$W_{\text {noise, in }}=W_{\mathrm{sq}}\left(x_{2}, p_{2}, x_{3}, p_{3}\right) W_{\mathrm{th}}\left(x_{2 t}, p_{2 t}\right) W_{\mathrm{th}}\left(x_{3 t}, p_{3 t}\right)$,

where

$W_{\text {th }}(x, p)=\frac{1}{\pi(\bar{n}+1 / 2)} e^{-\frac{1}{\bar{n}+1 / 2}\left(x^{2}+p^{2}\right)}$.

The action of the beam splitters corresponds to making the transformation

$$
\begin{aligned}
x_{2} & \rightarrow \sqrt{\eta_{2}} x_{2}+\sqrt{1-\eta_{2}} x_{2 t} \\
p_{2} & \rightarrow \sqrt{\eta_{2}} p_{2}+\sqrt{1-\eta_{2}} p_{2 t} \\
x_{2 t} & \rightarrow \sqrt{\eta_{2}} x_{2 t}-\sqrt{1-\eta_{2}} x_{2} \\
p_{2 t} & \rightarrow \sqrt{\eta_{2}} p_{2 t}-\sqrt{1-\eta_{2}} p_{2},
\end{aligned}
$$

in Eq. (11) together with a similar transformation for $x_{3}, p_{3}, x_{3 t}$, and $p_{3 t}$. The noise modes $\left(x_{2 t}, p_{2 t}\right)$ and $\left(x_{3 t}, p_{3 t}\right)$ are then traced out by integration. The resulting Wigner function describes the noisy two-mode squeezed input states for the teleportation protocol and is denoted $W_{s q}^{\text {in }}\left(x_{2}, p_{2}, x_{3}, p_{3}\right)$.

The probe state is in general described by a Gaussian Wigner function of the form

$W_{\mathrm{p}, i}^{\text {in }}\left(x_{1}, p_{1}\right)=c e^{-a_{x} x_{1}^{2}-\alpha_{p} p_{1}^{2}+\beta x_{1} p_{1}+\lambda_{x} x_{1}+\lambda_{p} p_{1}+\gamma}$.

where $c, a_{x}, a_{p}, \beta, \lambda_{x}, \lambda_{p}, y$ are constants. For the initial coherent state probe $\left(|-i a\rangle\right.$ with Wigner function $\left.W_{\mathrm{p}, 0}^{\text {in }}\right)$, we have that $c=\frac{2}{\pi}, a_{x}=a_{p}=2, \beta=0, \lambda_{x}=0, \lambda_{p}=-4 a, \gamma=-2 a^{2}$. The phase shift of the probe state makes the (unknown) transformation $x_{1} \rightarrow \cos (\phi) x_{1}+\sin$ $(\phi) p_{1}, p_{1} \rightarrow \cos (\phi) p_{1}-\sin (\phi) x_{1}$ in Eq. (14). Loss is modeled by subsequently mixing with a vacuum mode $\left(W_{\text {th }}\right.$ with $\left.\bar{n}=0\right)$ on a fictitious beam splitter of transmission $\eta_{1}$ and tracing out the output noise mode as with the twomode squeezed vacuum states. The resulting state is described by a Wigner function $W_{\mathrm{p}, i}^{\text {out }}\left(x_{1}, p_{1}\right)$.

The noisy two-mode squeezed input states, $W_{s q}^{\text {in }}\left(x_{2}, p_{2}, x_{3}, p_{3}\right)$ are now used to teleport the probe state $W_{\mathrm{p} i}^{\text {out }}\left(x_{1}, p_{1}\right)$ back to interact with the phase shift again. The mode $\left(x_{3}, p_{3}\right)$ is assumed to be the delayed mode and the goal is thus to teleport the mode $\left(x_{1}, p_{1}\right)$ back to this mode. This is done by mixing the modes $\left(x_{2}, p_{2}\right)$ and $\left(x_{1}, p_{1}\right)$ on a 50:50 beam splitter, measuring the output quadratures and providing feedback on the modes $\left(x_{3}, p_{3}\right)$. The input state before the 50:50 beam splitter has the Wigner function

$W_{\text {tel, in }}=W_{\mathrm{sq}}^{\text {in }}\left(x_{2}, p_{2}, x_{3}, p_{3}\right) W_{\mathrm{p}, i}^{\text {out }}\left(x_{1}, p_{1}\right)$.

The beam splitter makes the transformation

$x_{1} \rightarrow\left(x_{1}+x_{2}\right) / \sqrt{2}, \quad p_{1} \rightarrow\left(p_{1}+p_{2}\right) / \sqrt{2}$

$x_{2} \rightarrow\left(x_{1}-x_{2}\right) / \sqrt{2}, \quad p_{2} \rightarrow\left(p_{1}-p_{2}\right) / \sqrt{2}$

in Eq. (15). The measurements of the quadratures $p_{1}$ and $x_{2}$ and corresponding feedback on mode $\left(x_{3}, p_{3}\right)$ are then described by first making the transformation $x_{3} \rightarrow x_{3}-g_{x} \sqrt{2} x_{2}$, and $p_{3} \rightarrow p_{3}-p_{x} \sqrt{2} p_{2}$ in the Wigner function and then integrating out modes $\left(x_{1}, p_{1}\right)$ and $\left(x_{2}, p_{2}\right)$. The corresponding teleported state corresponds to probe state $W_{p, i+1}^{\text {in }}\left(x_{1}, p_{1}\right)$. The process can then be repeated to get the next iteration. After a fixed number of teleportations $m$, the phase shift is estimated from measurement of $\left\langle x_{1}\right\rangle$ for the light described by Wigner function $W_{0 m}^{\text {out }}\left(x_{1}, p_{1}\right)$. All moments of $x_{1}$ and $p_{1}$ can be extracted from $W_{\mathrm{p}, m}^{\text {out }}\left(x_{1}, p_{1}\right)$, which makes the calculation of the sensitivity straightforward. Note that the mean number of photons contained in the state is $n_{m}=\left\langle x_{1}^{2}\right\rangle+\left\langle p_{1}^{2}\right\rangle-1 / 2$.
Numerical optimization

For arbitrary gains $g_{x}$ and $g_{p}$, it becomes infeasible to find a closed analytical expression for the sensitivity for an arbitrary number of teleportations $\mathrm{m}$. For Figs. 2 and 3 , we have therefore numerically minimized the sensitivity in both the number of teleportations $(m)$, the gains $\left(g_{x}, g_{p}\right)$ (assumed fixed for each iteration), and $a$ for given values of the squeezing parameter $(r)$ and photon loss $\left(\eta_{1}, \eta_{2}\right)$. In the optimization, we use the analytical expressions for the teleported state derived as described above. By inserting the numerical values for the constants $\left(c, a_{x}\right.$ $\left.a_{p}, \beta, \lambda_{x}, \lambda_{p}, \gamma\right)$, we can repeatedly iterate the protocol and get the values of the constants for the final Wigner function $W_{\mathrm{p}, m}^{\text {out }}\left(x_{1}, p_{1}\right)$ after $m$ teleportations. From this, the sensitivity and the mean number of probe photons can be calculated. The optimal $m, g_{x}, g_{p}$ and $a$ are then identified as the ones that minimize the sensitivity under the constraint of a fixed number of total probe photons.

\section{DATA AVAILABILITY}

The datasets generated during and/or analyzed during the current study are available from the corresponding author on reasonable request.

\section{ACKNOWLEDGEMENTS}

We would like to thank Matthias Christandl for valuable feedback on the manuscript and helpful discussions. We also acknowledge funding from Center for Macroscopic Quantum States (bigQ DNRF142) and Qubiz - Quantum Innovation Center. J.B. acknowledges financial support from the European Research Council (ERC Grant Agreements no 337603), the Danish Council for Independent Research (Sapere Aude) VILLUM FONDEN via the QMATH Centre of Excellence (Grant No. 10059).

\section{AUTHOR CONTRIBUTIONS}

The project was conceived by J.B., T.G., J.S.N.-N. and U.L.A. The theory was developed by J.B. with details on experimental noise and considerations supplied by T.G., J.S.N.N., and U.L.A. All authors contributed in the writing of the manuscript.

\section{ADDITIONAL INFORMATION}

Competing interests: The authors declare no competing interests.

Publisher's note: Springer Nature remains neutral with regard to jurisdictional claims in published maps and institutional affiliations.

\section{REFERENCES}

1. Giovannetti, V., Lloyd, S. \& Maccone, L. Quantum-enhanced measurements: beating the standard quantum limit. Science 306, 1330-1336 (2004).

2. Giovannetti, V., Lloyd, S. \& Maccone, L. Advances in quantum metrology. Nat. Photonics 5, 222-229 (2011).

3. Escher, B. M., de Matos Filho, R. L. \& Davidovich, L. General framework for estimating the ultimate precision limit in noisy quantum-enhanced metrology. Nat. Phys. 7, 406-411 (2011).

4. Degen, C. L., Reinhard, F. \& Cappellaro, P. Quantum sensing. Rev. Mod. Phys. 89, 035002 (2017).

5. Pan, J.-W. et al. Multiphoton entanglement and interferometry. Rev. Mod. Phys. 84, 777-838 (2012).

6. Demkowicz-Dobrzański, R., Jarzyna, M. \& Kołodyński, J. Chapter four - quantum limits in optical interferometry. Progress. Opt. 60, 345-435 (2015).

7. Bouchard, F. et al. Quantum metrology at the limit with extremal majorana constellations. Optica 4, 1429-1432 (2017).

8. Frigault, M. M., Lacoste, J., Swift, J. L. \& Brown, C. M. Live-cell microscopy - tips and tools. J. Cell Sci. 122, 753-767 (2009).

9. Crespi, A. et al. Measuring protein concentration with entangled photons. Appl. Phys. Lett. 100, 233704 (2012).

10. Piliarik, M. \& Sandoghdar, V. Direct optical sensing of single unlabelled proteins and super-resolution imaging of their binding sites. Nat. Commun. 5, 4495 (2014).

11. Wolfgramm, F., Vitelli, C., Beduini, F. A., Godbout, N. \& Mitchell, M. W. Entanglement-enhanced probing of a delicate material system. Nat. Photonics 7, 28-32 (2012)

12. Mitchell, M. W., Lundeen, J. S. \& Steinberg, A. M. Super-resolving phase measurements with a multiphoton entangled state. Nature 429, 161-164 (2004).

13. Afek, I., Ambar, O. \& Silberberg, Y. High-noon states by mixing quantum and classical light. Science 328, 879-881 (2010). 
14. Facon, A. et al. A sensitive electrometer based on a rydberg atom in a schrödinger-cat state. Nature 535, 262-265 (2016).

15. Caves, C. M. Quantum-mechanical noise in an interferometer. Phys. Rev. D. 23, 1693-1708 (1981)

16. Collaboration, T. L. S. A gravitational wave observatory operating beyond the quantum shot-noise limit. Nat. Phys. 7, 962-965 (2011).

17. Aasi, J. et al. Enhanced sensitivity of the ligo gravitational wave detector by using squeezed states of light. Nat. Photonics 7, 613-619 (2013).

18. Taylor, M. A. et al. Biological measurement beyond the quantum limit. Nat. Photonics 7, 229-233 (2013).

19. Berni, A. A. et al. Ab initio quantum-enhanced optical phase estimation using real-time feedback control. Nat. Photonics 9, 577-581 (2015).

20. Schäfermeier, C. et al. Quantum enhanced feedback cooling of a mechanical oscillator using nonclassical light. Nat. Commun. 7, 13628 (2016).

21. Resch, K. J. et al. Time-reversal and super-resolving phase measurements. Phys. Rev. Lett. 98, 223601 (2007).

22. Giovannetti, V., Lloyd, S. \& Maccone, L. Quantum metrology. Phys. Rev. Lett. 96, 010401 (2006)

23. van Dam, W., D'Ariano, G. M., Ekert, A., Macchiavello, C. \& Mosca, M. Optimal quantum circuits for general phase estimation. Phys. Rev. Lett. 98, 090501 (2007).

24. Demkowicz-Dobrzański, R. Multi-pass classical vs. quantum strategies in lossy phase estimation. Laser Phys. 20, 1197-1202 (2010).

25. Higgins, B. L., Berry, D. W., Bartlett, S. D., Wiseman, H. M. \& Pryde, G. J. Entanglement-free heisenberg-limited phase estimation. Nature 450, 393-396 (2007).

26. Juffmann, T., Klopfer, B. B., Frankort, T. L. I., Haslinger, P. \& Kasevich, M. A. Multipass microscopy. Nat. Commun. 7, 12858 (2016).

27. Furusawa, A. et al. Unconditional quantum teleportation. Science 282, 706-709 (1998).

28. Yokoyama, S. et al. Ultra-large-scale continuous-variable cluster states multiplexed in the time domain. Nat. Photonics 7, 982-986 (2013).

29. Braunstein, S. L. \& Kimble, H. J. Teleportation of continuous quantum variables. Phys. Rev. Lett. 80, 869-872 (1998).
30. Bondurant, R. S. \& Shapiro, J. H. Squeezed states in phase-sensing interferometers. Phys. Rev. D 30, 2548-2556 (1984).

31. Xiang, G. Y., Higgins, B. L., Berry, D. W., Wiseman, H. M. \& Pryde, G. J. Entanglement-enhanced measurement of a completely unknown optical phase. Nat. Photonics 5, 43-47 (2010).

32. Mitchell, M. W. Metrology with entangled states. Proc. SPIE 5893 (2005). https:// doi.org/10.1117/12.621353.

33. Kessler, E. M. et al. Heisenberg-limited atom clocks based on entangled qubits. Phys. Rev. Lett. 112, 190403 (2014).

34. Sokolov, I., Kolobov, M., Gatti, A. \& Lugiato, L. Quantum holographic teleportation. Opt. Commun. 193, 175-180 (2001).

35. Seshadreesan, K. P., Dowling, J. P. \& Agarwal, G. S. Non-gaussian entangled states and quantum teleportation of schrödinger-cat states. Phys. Scr. 7, 074029 (2015).

36. Lindner, N. H. \& Rudolph, T. Proposal for pulsed on-demand sources of photonic cluster state strings. Phys. Rev. Lett. 103, 113602 (2009).

37. Gerry, C. \& Knight, P. Introductory Quantum Optics (Cambridge University Press, New York, 2005).

(i) Open Access This article is licensed under a Creative Commons Attribution 4.0 International License, which permits use, sharing, adaptation, distribution and reproduction in any medium or format, as long as you give appropriate credit to the original author(s) and the source, provide a link to the Creative Commons license, and indicate if changes were made. The images or other third party material in this article are included in the article's Creative Commons license, unless indicated otherwise in a credit line to the material. If material is not included in the article's Creative Commons license and your intended use is not permitted by statutory regulation or exceeds the permitted use, you will need to obtain permission directly from the copyright holder. To view a copy of this license, visit http://creativecommons. org/licenses/by/4.0/.

(c) The Author(s) 2019 